\title{
OUTPUT ERROR METHOD FOR TILTROTOR UNSTABLE IN HOVER
}

\begin{abstract}
This article investigates unstable tiltrotor in hover system identification from flight test data. The aircraft dynamics was described by a linear model defined in Body-FixedCoordinate System. Output Error Method was selected in order to obtain stability and control derivatives in lateral motion. For estimating model parameters both time and frequency domain formulations were applied. To improve the system identification performed in the time domain, a stabilization matrix was included for evaluating the states. In the end, estimates obtained from various Output Error Method formulations were compared in terms of parameters accuracy and time histories. Evaluations were performed in MATLAB R2009b environment.
\end{abstract}

\section{Introduction}

Modern military aviation has to face many problems, among which increasing performance is one of the highest interests. This fact leads to the development of tiltrotors - vertical take-off and landing aircrafts that can also carry the load at a higher speed than conventional helicopters. In order to achieve this aim, two rotors are mounted on a rotating nacelles that are located on the end of a fixed wing. Due to this design, the aircraft can operate like a helicopter - when the rotors are angled so that the rotation plane is horizontal, or like a turboprop aircraft - when the rotation plane is vertical.

When a tiltrotor is in hover, it experiences the same problem as a conventional helicopter that is mode instability e.g. Bo-105 [1]. To make the aircraft possible to fly by the pilot under those conditions, a feedback control is added so that the system operates in the closed loop [2]. In the case of a sensor failure, the control laws must be modified so the system has to be identified earlier in an open loop. Moreover, the open loop estimation makes it possible to validate the model

\footnotetext{
${ }^{1}$ Warsaw University of Technology, Institute of Aeronautics and Applied Mechanics, Poland. Emails: plichota@meil.pw.edu.pl, jszulczyk@meil.pw.edu.pl
} 
parameters obtained from flight test data by comparing them with wind tunnel test data or to extend the flight envelope. This is also true for high manoeuvrable aircrafts that are often designed as unstable, or have relaxed static stability, in order to improve their combat performance e.g. X-29 [3], X-31A [4], Eurofighter [5], as well as unmanned aerial vehicles e.g. helicopter-based Yamaha R-50 [6], Ikarus $\mathrm{ECO}$ [7] or even more complex aircrafts that recently catch a great deal of interest, such as Quadcopters [8] and Cyclocopters [9].

Output Error Method (OEM) is the most frequently used System Identification (Sys-ID) technique. It represents a natural formulation for a dynamic system and allows one to obtain high-accuracy estimates of the model parameters if there is enough information in the measured data and the process noise is negligible. The main drawback of the OEM is that it requires a priori information for the unknown model parameters. Usually, this is not a serious limitation as the initial values of the aerodynamic coefficients can be obtained from various sources e.g. engineering methods, wind tunnel tests or Computational Fluid Dynamics [10].

Another drawback of the OEM is its application for unstable systems parameter estimation. This deficiency comes rather from the numerical issues than the theoretical formulation, as it is required to integrate equations that are sensible to the initial conditions and model parameters. Therefore, the estimation process can easily diverge leading to unrealistic values of the aerodynamic coefficients. To overcome this difficulty for the time domain approach, an artificial stabilization matrix can be included in the model equations. Another option is to perform the parameter estimation in the frequency domain. In this article, we present both approaches and compare them with Sys-ID performed in the time domain for a tiltrotor in hover.

The rest of this paper is structured as follows. First, we describe the real object and develop its mathematical model. Then, we give an overview of the Sys-ID and OEM techniques. This section is divided into three parts - each of them describes a different OEM approach. Firstly, we describe the traditional time domain formulation. In the second part, an artificial stabilization is introduced. The last part presents the frequency domain formulation. In the next section of the paper we present the results obtained by using various OEM approaches and real flight test data. The parameters accuracy and time history plots are used to compare the OEM techniques that we used for the tiltrotor Sys-ID. Finally, a brief summary of conclusions is given.

\section{Mathematical model}

The test aircraft used in this study was the XV-15 - a two-pilot tiltrotor that has a wingspan of $17.42 \mathrm{~m}$, weights $4574 \mathrm{~kg}$ (MEW) and is powered by two turboshaft engines producing $1156 \mathrm{~kW}$ each. The dynamic response of the object was obtained from [11] for the manoeuvres in which frequency sweep and doublet signals were used to excite the motion. The flight data set consisted of roll rate $p$, yaw rate $r$, bank angle $\phi$, lateral acceleration $a_{y}$, ailerons $\delta_{A}$ and rudder $\delta_{R}$ 
deflections measurements. All signals were sampled at $250 \mathrm{~Hz}$ what is much more than usually required for aircraft Sys-ID $(50 \mathrm{~Hz})$.

In order to develop the mathematical model of the XV-15 tiltrotor, we used the typical flight dynamics assumptions. The aircraft was modelled as a rigid body with moveable flight controls and a vertical symmetry plane. As each manoeuvre lasts less than $100 \mathrm{~s}$, the mass of the object and gravitational acceleration were considered constant, and flat Earth approximation was used. For aerodynamic modelling, it was assumed that flight parameters have linear influence on aerodynamic forces and moments. The last assumption was introduced also in order to compare various OEM approaches as frequency domain allows us to identify only linear systems.

The tiltrotor equations of motion were derived in an orthogonal Body-fixed coordinate system $O x y z$. The origin of the reference frame was located at aircraft centre of mass and the longitudinal axis $O x$ was parallel to the mean aerodynamic chord. The lateral axis $O y$ was directed towards right wing whilst the vertical axis $O z$ completed the right-handed set. The reference frame $O x y z$ was related to a vehicle-carried coordinate system $O x_{g} y_{g} z_{g}$ that is always parallel to a non-rotating Earth-fixed frame $O_{1} x_{1} y_{1} z_{1}$ through the attitude angles (bank $\phi$, pitch $\theta$ and yaw $\psi)$. This relation is presented in Fig. 1.

The linear $\boldsymbol{\Lambda}_{V}$ and angular quantities transformations $\boldsymbol{\Lambda}_{\Omega}$ between $O x y z$ and $O x_{g} y_{g} z_{g}$ are given by the following matrices [12]:

$$
\begin{gathered}
\boldsymbol{\Lambda}_{V}=\left[\begin{array}{ccc}
1 & 0 & 0 \\
0 & \cos \Phi & \sin \Phi \\
0 & -\sin \Phi & \cos \Phi
\end{array}\right]\left[\begin{array}{ccc}
\cos \Theta & 0 & -\sin \Theta \\
0 & 1 & 0 \\
\sin \Theta & 0 & \cos \Theta
\end{array}\right]\left[\begin{array}{ccc}
\cos \Psi & \sin \Psi & 0 \\
-\sin \Psi & \cos \Psi & 0 \\
0 & 0 & 1
\end{array}\right] \\
\Lambda_{\Omega}=\left[\begin{array}{ccc}
1 & 0 & -\sin \Theta \\
0 & \cos \Phi & \sin \Phi \cos \Theta \\
0 & -\sin \Phi & \cos \Phi \cos \Theta
\end{array}\right]
\end{gathered}
$$

The equations of motion were derived from momentum $\boldsymbol{\Pi}$ and angular momentum $\mathbf{K}_{O}$ change theorems:

$$
\begin{gathered}
\frac{\delta \boldsymbol{\Pi}}{\delta t}+\mathbf{\Omega} \times \mathbf{\Pi}=\mathbf{F} \\
\frac{\delta \mathbf{K}_{O}}{\delta t}+\mathbf{\Omega} \times \mathbf{K}_{O}=\mathbf{M}_{O}
\end{gathered}
$$

where $\mathbf{F}=\left[\begin{array}{lll}X & Y & Z\end{array}\right]^{T}$ and $\mathbf{M}_{O}=\left[\begin{array}{lll}L & M & N\end{array}\right]^{T}$ stand for external force and moment, $\mathbf{\Omega}=\left[\begin{array}{lll}P & Q & R\end{array}\right]^{T}$ is the angular velocity and $\delta / \delta t$ denotes a local derivative. 


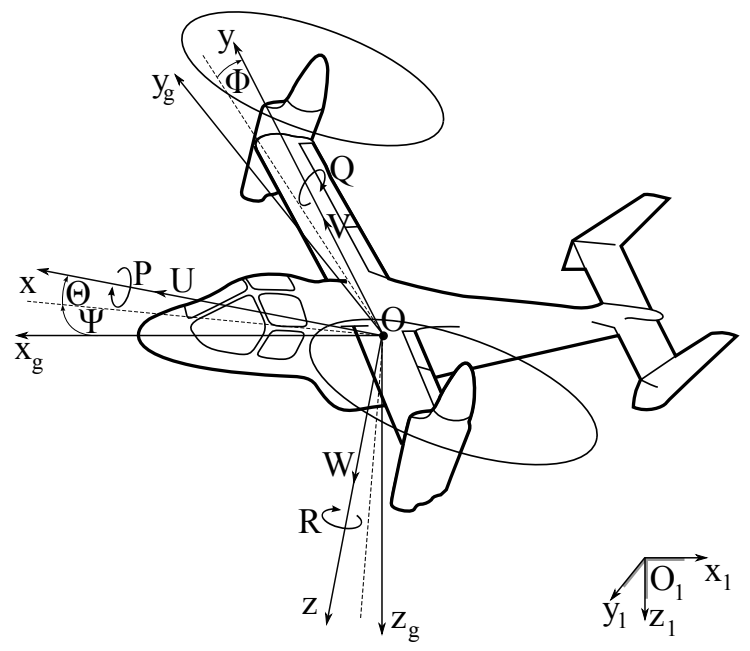

Fig. 1. Coordinate systems

Adding kinematic relationships to the above equations leads to:

$$
\begin{gathered}
m\left(\dot{\boldsymbol{V}}_{O}+\mathbf{\Omega} \times \mathbf{V}_{O}\right)=\mathbf{F} \\
\mathbf{I} \dot{\boldsymbol{\Omega}}+\mathbf{\Omega} \times(\mathbf{I} \boldsymbol{\Omega})=\mathbf{M}_{O} \\
\dot{\boldsymbol{\Phi}}=\boldsymbol{\Lambda}_{\Omega}^{-1} \mathbf{\Omega}
\end{gathered}
$$

where $\mathbf{V}_{O}=\left[\begin{array}{lll}U & V & W\end{array}\right]^{T}$ is the linear velocity, $\dot{\boldsymbol{\Phi}}=\left[\begin{array}{lll}\dot{\Phi} & \dot{\Theta} & \dot{\Psi}\end{array}\right]^{T}$ describes the aircraft orientation, $m$ and $\mathbf{I}$ stand for mass and inertia tensor whilst the dot symbol denotes a derivative with respect to time.

Applying the small perturbation theorem allows one to linearize Eq. (3). Moreover, as only the lateral motion parameters are to be estimated and in the trim conditions, all the flight parameters have zero values the system can be described by the following state equation:

$$
\left[\begin{array}{c}
\dot{V} \\
\dot{P} \\
\dot{R} \\
\dot{\phi}
\end{array}\right]=\left[\begin{array}{cccc}
Y_{v} & Y_{p} & Y_{r} & g \\
L_{v} & L_{p} & L_{r} & 0 \\
N_{v} & N_{p} & N_{r} & 0 \\
0 & 1 & 0 & 0
\end{array}\right]\left[\begin{array}{c}
V \\
P \\
R \\
\phi
\end{array}\right]+\left[\begin{array}{cc}
Y_{\delta_{A}} & Y_{\delta_{R}} \\
L_{\delta_{A}} & L_{\delta_{R}} \\
N_{\delta_{A}} & N_{\delta_{R}} \\
0 & 0
\end{array}\right]\left[\begin{array}{c}
\delta_{A} \\
\delta_{R}
\end{array}\right]
$$

where $V$ is the lateral velocity, $P$ and $R$ are roll and yaw rates, $\phi$ is the bank angle, $\delta_{A}$ and $\delta_{R}$ are the aileron and rudder deflection and $g$ stands for gravitational acceleration. The rest of the terms in Eq (4) is known as stability and control derivatives. 
Lateral acceleration is added to the output equation in order to increase the accuracy of the estimation:

$$
\left[\begin{array}{c}
V \\
P \\
R \\
\phi \\
a_{y}
\end{array}\right]=\left[\begin{array}{cccc}
1 & 0 & 0 & 0 \\
0 & 1 & 0 & 0 \\
0 & 0 & 1 & 0 \\
0 & 0 & 0 & 1 \\
Y_{v} & Y_{p} & Y_{r} & 0
\end{array}\right]\left[\begin{array}{c}
V \\
P \\
R \\
\phi
\end{array}\right]+\left[\begin{array}{cc}
0 & 0 \\
0 & 0 \\
0 & 0 \\
0 & 0 \\
Y_{\delta_{A}} & Y_{\delta_{R}}
\end{array}\right]\left[\begin{array}{l}
\delta_{A} \\
\delta_{R}
\end{array}\right]
$$

The side-force $Y$, roll $L$ and yaw moment $N$ derivatives in Eq. (4) and Eq. (5) with respect to a $j$-th flight parameter are defined as:

$$
\begin{gathered}
Y_{j}=\frac{1}{m} \frac{\partial Y}{\partial j} \\
L_{j}=\frac{I_{z z}}{I_{x x} I_{z z}-I_{x z}^{2}} \frac{\partial L}{\partial j}+\frac{I_{x z}}{I_{x x} I_{z z}-I_{x z}^{2}} \frac{\partial N}{\partial j} \\
N_{j}=\frac{I_{x z}}{I_{x x} I_{z z}-I_{x z}^{2}} \frac{\partial N}{\partial j}+\frac{I_{x x}}{I_{x x} I_{z z}-I_{x z}^{2}} \frac{\partial L}{\partial j}
\end{gathered}
$$

\section{System Identification}

The stability and control derivatives of the XV-15 aircraft were obtained from the flight test data by performing the Sys-ID that can be described as "the determination, on the basis on input and output, of a system within a specified class of systems, to which the system under test is equivalent" [13]. This means that the structure of the mathematical model and its parameters $\boldsymbol{\Theta}$ (aerodynamic derivatives) were adjusted so that for the same inputs $\mathbf{u}$ the model outputs $\mathbf{y}$ match the measured aircraft response $\mathbf{z}$ [14]. However, due to many reasons (e.g. measurement noise, secondary effects that were not modelled, etc.) it was impossible to obtain an ideal match between the flight test data and the model outputs. Sys-ID was performed in the offline mode because the data had been gathered prior to parameter estimation. The scheme of the OEM is presented in Fig. 2.

To perform the aircraft Sys-ID, multiple different methods can be used i.e. Equation Error, Output Error, Filter Error or Artificial Neural Networks. Among this group, the OEM is the most popular because it represents a natural formulation for a dynamic system allowing a great flexibility in the model structure. The main drawback of the OEM is that it cannot be used when a high process noise (e.g. due to turbulence) is observed in the data. However, for aircraft Sys-ID usually this is not a serious limitation, as the flight tests are performed in the good weather conditions. In the next subsections, we present various OEM formulations based on Maximum Likelihood Principle: the conventional time domain approach, the 


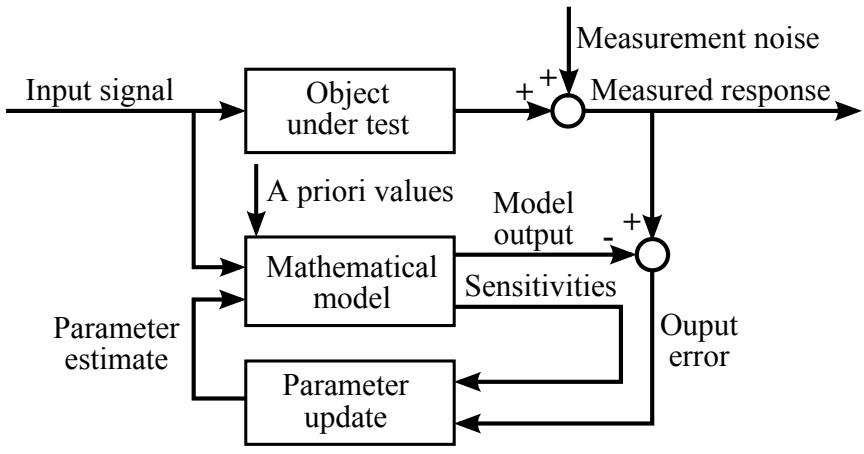

Fig. 2. Output Error Method block diagram

time domain technique with artificial stabilization matrix for improving numerical performance and the frequency domain formulation.

\subsection{Time Domain}

In general, a dynamic system can be described by the following equations [15]:

$$
\begin{gathered}
\dot{\mathbf{x}}(t)=f(\mathbf{x}(t), \mathbf{u}(t), \boldsymbol{\Theta}) \quad \mathbf{x}(t=0)=\mathbf{x}_{0} \\
\mathbf{y}(t)=g(\mathbf{x}(t), \mathbf{u}(t), \boldsymbol{\Theta}) \\
\mathbf{z}\left(t_{k}\right)=\mathbf{y}\left(t_{k}\right)+\boldsymbol{v}\left(t_{k}\right)
\end{gathered}
$$

where $\mathbf{u}, \mathbf{x}, \mathbf{y}, \mathbf{z}$ denote inputs, states, model outputs and measured response, respectively, whilst $\boldsymbol{v}$ stand for measurement error, $\boldsymbol{\Theta}$ are model parameters and $t_{k}$ is the discrete time at $k$-th point, $k=1, \ldots, N$. General state and output functions are denoted as $f$ and $g$.

Obtaining the parameter estimates for the following system through OEM by applying Maximum Likelihood Principle in the time domain requires finding model parameters that maximize the probability of observing the measured response:

$$
\hat{\boldsymbol{\Theta}}=\arg \max _{\boldsymbol{\Theta}} p(\mathbf{z} \mid \boldsymbol{\Theta})
$$

where hat symbol denotes the estimates.

Selecting multivariable Gaussian distribution allows one to define the probability for all recorded time points [16, 17]:

$$
p(\mathbf{z} \mid \Theta)=\frac{1}{{\sqrt{(2 \pi)^{n}|\mathbf{R}|}}^{N}} \exp \left(-\frac{1}{2} \sum_{k=1}^{N}\left[\mathbf{z}\left(t_{k}\right)-\mathbf{y}\left(t_{k}\right)\right]^{T} \mathbf{R}^{-1}\left[\mathbf{z}\left(t_{k}\right)-\mathbf{y}\left(t_{k}\right)\right]\right)
$$

where $\mathbf{R}$ is the measurement noise covariance matrix and $n$ denotes the number of model outputs. 
Maximization of the probability given by Eq. (9) is usually replaced by minimization of the corresponding negative log-likelihood function:

$$
\mathcal{L}(\boldsymbol{\Theta} \mid \mathbf{z})=\frac{1}{2} \sum_{k=1}^{N}\left[\mathbf{z}\left(t_{k}\right)-\mathbf{y}\left(t_{k}\right)\right]^{T} \mathbf{R}^{-1}\left[\mathbf{z}\left(t_{k}\right)-\mathbf{y}\left(t_{k}\right)\right]+\frac{n N}{2} \ln (2 \pi)+\frac{N}{2} \ln (\operatorname{det}(\mathbf{R}))
$$

and the unknown measurement covariance matrix $\mathbf{R}$ is estimated as:

$$
\hat{\mathbf{R}}=\frac{1}{N} \sum_{k=1}^{N}\left[\mathbf{z}\left(t_{k}\right)-\mathbf{y}\left(t_{k}\right)\right]\left[\mathbf{z}\left(t_{k}\right)-\mathbf{y}\left(t_{k}\right)\right]^{T}
$$

After substituting Eq. (11) into Eq. (10), neglecting constant terms and assuming that measurement errors are uncorrelated the final cost function $J(\boldsymbol{\Theta})$ is given by:

$$
J(\boldsymbol{\Theta})=\prod_{i=1}^{m} \frac{1}{N} \sum_{k=1}^{N}\left(z_{i}\left(t_{k}\right)-y_{i}\left(t_{k}\right)\right)^{2}
$$

where $i=1, \ldots m$ is the number of system outputs.

As it can be seen, the OEM leads to a nonlinear estimation problem that requires a multi-dimensional optimization method to find the solution. It requires the a priori knowledge of the estimated parameters, and in the case of poor initial guess it can lead to excessive iterations and incorrect values of the aerodynamic derivatives. As the time-domain formulation requires states integration, this problem can be even more crucial because incorrect values of the estimated parameters can easily lead to a diverged solution.

\subsection{Time Domain with Artificial Stabilization}

To overcome the problems with the states integration in the OEM time domain approach, an artificial stabilization matrix $\mathbf{S}$ can be used. If this technique is applied, the states are updated according to the following equation:

$$
\mathbf{x}\left(t_{k}\right)=\mathbf{x}\left(t_{k}\right)+\mathbf{S}\left[\mathbf{z}\left(t_{k}\right)-\mathbf{y}\left(t_{k}\right)\right]
$$

From the Eq. (13) it can be observed that, for the case when the artificial stabilization is a null matrix, the formulation is equivalent to the conventional OEM time domain formulation. However, as the stabilization matrix is introduced to improve the OEM time domain stability it is arbitrarily selected depending on the system behaviour knowledge (aerodynamic parameters influence) and measurements reliability.

It has to be mentioned that the artificial stabilization introduce errors in the parameter estimates. Therefore, the elements of the matrix should have small values, because in this case those modelling errors will be small and will not affect the parameter estimates significantly. 
Besides mentioned features, the stabilized approach has the same properties as the conventional time-domain formulation. This means that it can be used also for a nonlinear object Sys-ID when the time-domain approach fails due to states integration problems.

\subsection{Frequency Domain}

In order to perform the Sys-ID in the frequency domain, measured data must be transformed by using the finite Fourier transform that can be approximated by $[18,19]$ :

$$
\tilde{x}(f) \approx \Delta t \sum_{k=1}^{N} x\left(t_{k}\right) \exp (-i 2 \pi f k \Delta t)
$$

where $f$ is the frequency of a measured discrete signal $x$ that is evenly spaced at $\Delta t$ time intervals, $i$ stands for the imaginary unit $i=\sqrt{-1}$ and tilde symbol denotes the transformed signal. The evaluations in the frequency domain are performed at discrete frequencies $f_{j}=j /(N \Delta t)$. Therefore, for a unit sampling interval the measured signals can be transformed between time and frequency domains by using Discrete Fourier Transform [19]:

$$
X\left(f_{j}\right)=\sum_{k=1}^{N} x\left(t_{k}\right) \exp \left(-i 2 \pi \frac{j k}{N}\right)
$$

Performing those computations is not the most efficient way, because the frequencies are fixed and evenly spaced. To select different frequencies, a zero-padding can be done but this approach interpolates the frequency domain data rather than increases its resolution. To overcome this problem, a Chirp Z-Transform [20] can be used.

When the Eq. (15) is written as:

$$
\begin{gathered}
X\left(f_{j}\right)=\sum_{k=1}^{N} x\left(t_{k}\right) A W^{-j k} \\
A=1 \\
W=\exp \left(i \frac{2 \pi}{N}\right)
\end{gathered}
$$

it can be viewed in the complex plane as a unit circle with equally spaced frequencies at $2 \pi j / N$. Modifying $A$ allows us to start the contour at lower limit of frequency range of interest $\omega_{\min }$ and adjusting $W$ makes it possible to select specific (e.g. uniformly spaced) frequencies $\Delta \omega$ :

$$
\begin{gathered}
A=\exp \left(i \omega_{\min } \Delta t\right) \\
W=\exp (i \Delta \omega \Delta t)
\end{gathered}
$$


This way of restricting frequencies number allows one to reduce the amount of evaluated data points significantly. Omitting lowest frequencies allows to eliminate biases from the model structure leading to fewer estimates. Neglecting higher frequencies filters measured signals and eliminates higher-order dynamics [21]. Regarding the OEM in the frequency domain, it should be also highlighted that for aircraft Sys-ID frequency points are usually linearly spaced in comparison to the time-domain formulation where the lowest frequencies have the most time samples and therefore influence the cost function the most.

When the measured signals are transformed into frequency domain, the object is described by the following equations:

$$
\begin{gathered}
i \omega_{j} \tilde{\mathbf{x}}\left(\omega_{j}\right)=\mathbf{A} \tilde{\mathbf{x}}\left(\omega_{j}\right)+\mathbf{B} \tilde{\mathbf{u}}\left(\omega_{j}\right) \\
\tilde{\mathbf{y}}\left(\omega_{j}\right)=\mathbf{C} \tilde{\mathbf{x}}\left(\omega_{j}\right)+\mathbf{D} \tilde{\mathbf{u}}\left(\omega_{j}\right) \\
\tilde{\mathbf{z}}\left(\omega_{j}\right)=\tilde{\mathbf{y}}\left(\omega_{j}\right)+\tilde{\boldsymbol{v}}\left(\omega_{j}\right)
\end{gathered}
$$

From Eq. (7) and Eq. (18) it can be observed that, in contrast to the time-domain approach, the frequency domain allows to estimate only linear systems what is the greatest deficiency of this formulation. The only way in which nonlinear terms can be included is to evaluate those terms using measured signals and use them as additional inputs. On the other hand, this approach does not require integrating the states what makes this representation useful for unstable systems parameter estimation.

Applying Maximum Likelihood principle results in a negative log-likelihood function derived analogously to its time domain formulation given in Eq. (10):

$\mathcal{L}(\boldsymbol{\Theta} \mid \tilde{\mathbf{z}})=\frac{1}{2} \sum_{k=1}^{N}\left[\tilde{\mathbf{z}}\left(\omega_{j}\right)-\tilde{\mathbf{y}}\left(\omega_{j}\right)\right]^{\dagger} \mathbf{S}^{-1}\left[\tilde{\mathbf{z}}\left(\omega_{j}\right)-\tilde{\mathbf{y}}\left(\omega_{j}\right)\right]+\frac{n N}{2} \ln (2 \pi)+\frac{N}{2} \ln (\operatorname{det}(\mathbf{S}))$

where $\dagger$ denotes conjugate transpose of a complex matrix and $\mathbf{S}$ is the power spectral density of the measurement noise that can be estimated from:

$$
\hat{\mathbf{S}}=\frac{1}{N} \sum_{j=1}^{N}\left[\tilde{\mathbf{z}}\left(\omega_{j}\right)-\tilde{\mathbf{y}}\left(\omega_{j}\right)\right]\left[\tilde{\mathbf{z}}\left(\omega_{j}\right)-\tilde{\mathbf{y}}\left(\omega_{j}\right)\right]^{\dagger}
$$

Similarly to the negative log-likelihood, the final cost function is analogous to its time domain formulation given in Eq. (12):

$$
J(\Theta)=\prod_{i=1}^{m} \frac{1}{N} \sum_{j=1}^{N}\left(\tilde{z}_{i}\left(\omega_{j}\right)-\tilde{y}_{i}\left(\omega_{j}\right)\right)^{\dagger}\left(\tilde{z}_{i}\left(\omega_{j}\right)-\tilde{y}_{i}\left(\omega_{j}\right)\right)
$$




\section{Results}

Due to frequency domain limitations, the system was identified as a secondorder model. Recorded roll rate $P$, yaw rate $R$, bank angle $\phi$, lateral acceleration $a_{y}$, aileron $\delta_{A}$ and rudder deflections $\delta_{R}$ were used for that purpose. The lateral velocity was not recorded, and therefore was not weighted in the cost function given by Eq. (12) or Eq. (21). The signals were sampled at $250 \mathrm{~Hz}$.

When the aircraft is modelled as a rigid body, a $50 \mathrm{~Hz}$ sampling is used to cover the frequency range of interest. As the recorded signals were sampled at $250 \mathrm{~Hz}$ it was found necessary to smooth them by a low pass digital filter in order to remove unmodelled effects (e.g. engine dynamics) from the data.

To minimize the cost function, Gauss-Newton algorithm was used. In the time domain the parameter updates $\Delta \boldsymbol{\Theta}$ were evaluated according to the equation:

$$
\underbrace{\sum_{k=1}^{N}\left[\frac{\partial \mathbf{y}\left(t_{k}\right)}{\partial \boldsymbol{\Theta}}\right]^{T} \mathbf{R}^{-1}\left[\frac{\partial \mathbf{y}\left(t_{k}\right)}{\partial \boldsymbol{\Theta}}\right]}_{\mathcal{F}} \Delta \boldsymbol{\Theta}=\sum_{k=1}^{N}\left[\frac{\partial \mathbf{y}\left(t_{k}\right)}{\partial \boldsymbol{\Theta}}\right]^{T} \mathbf{R}^{-1}\left[\mathbf{z}\left(t_{k}\right)-\mathbf{y}\left(t_{k}\right)\right]
$$

where $\mathcal{F}$ is known as the Fisher Information Matrix. Analogous formula was used in the frequency domain

$$
\underbrace{\sum_{j=1}^{N}\left[\frac{\partial \tilde{\mathbf{y}}\left(\omega_{j}\right)}{\partial \boldsymbol{\Theta}}\right]^{\dagger} \mathbf{S}^{-1}\left[\frac{\partial \tilde{\mathbf{y}}\left(\omega_{j}\right)}{\partial \boldsymbol{\Theta}}\right]}_{\mathcal{F}} \Delta \boldsymbol{\Theta}=\sum_{j=1}^{N}\left[\frac{\partial \tilde{\mathbf{y}}\left(\omega_{j}\right)}{\partial \boldsymbol{\Theta}}\right]^{\dagger} \mathbf{S}^{-1}\left[\tilde{\mathbf{z}}\left(\omega_{j}\right)-\tilde{\mathbf{y}}\left(\omega_{j}\right)\right]
$$

In order to improve the Gauss-Newton algorithm convergence, the parameters were updated according to the formula:

$$
\boldsymbol{\Theta}_{i+1}=\boldsymbol{\Theta}_{i}+\alpha_{i} \Delta \boldsymbol{\Theta}
$$

where $i$ subscript denotes the iteration number. In each iteration, the final scaling factor $\alpha_{i}$ was based on the cost function values evaluated for three different scaling factors. Through the points specified by the scaling factors and the corresponding cost function values, a second order function has been fitted and the final scaling factor was located at its minimum.

Sys-ID results that were obtained using various OEM approaches are presented in Fig. 3 and Table 1. In the plot, green, red and black dashed lines represent model outputs from the time domain, time domain with artificial stabilization and frequency domain whilst the blue solid line represents the measurement. The stability and control derivatives in the table are defined with respect to SI units and radians. The relative standard deviations represent the parameters accuracy. Standard deviations were obtained from the elements of the Fisher Information Matrix inverse:

$$
\sigma\left(\boldsymbol{\Theta}_{i}\right)=\sqrt{\mathcal{F}_{i i}^{-1}}
$$



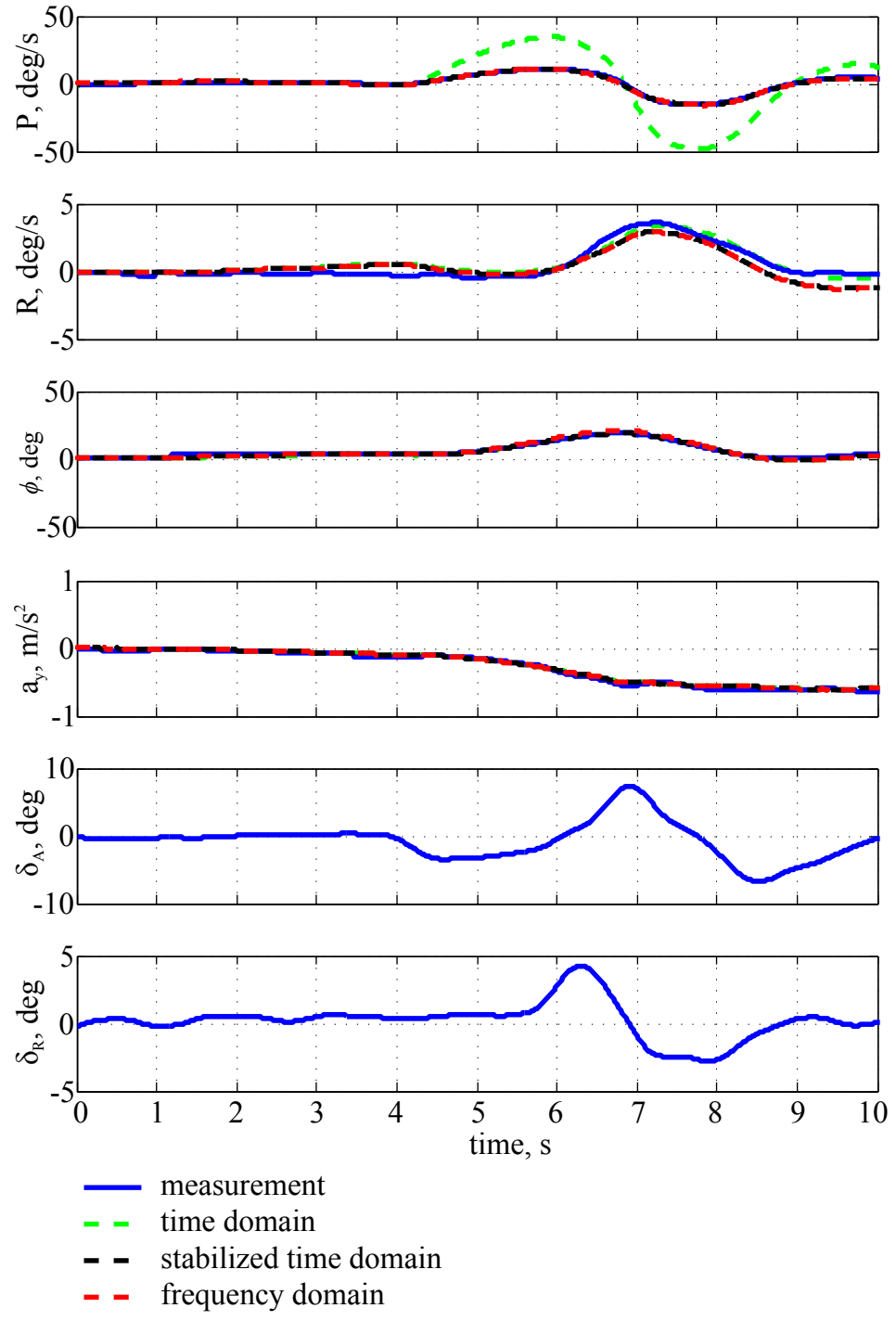

Fig. 3. Time history plots

From the time history plots it can be observed that, except the roll rate, the time domain approach allowed us to obtain a good match between the model outputs and measurements. The artificial stabilization made it possible to improve the match between the curves, and small mismatch can be observed only for the yaw rate. This is true also for the frequency domain approach for which the model outputs visually look very similar to the ones obtained for the stabilized time domain formulation.

Similar conclusions come from Table 1. The relative standard deviations were the smallest for the frequency domain and stabilized time-domain approaches. 
Estimated stability and control derivatives

\begin{tabular}{|c|r|r|r|c|c|c|}
\hline \multirow{2}{*}{ Name } & \multicolumn{6}{|c|}{ Method } \\
\cline { 2 - 7 } & \multicolumn{1}{|c|}{ Time Domain } & \multicolumn{1}{|c|}{ Stab. Time Domain } & \multicolumn{2}{c|}{ Frequency Domain } \\
\cline { 2 - 7 } & \multicolumn{1}{|c}{$\hat{\boldsymbol{\Theta}}$} & $\sigma_{\text {rel }}, \%$ & \multicolumn{1}{|c}{} & $\sigma_{\text {rel }}, \%$ & $\hat{\boldsymbol{\Theta}}$ & $\sigma_{r e l}, \%$ \\
\hline$Y_{v}$ & -0.1698 & 17.82 & -0.0913 & 0.49 & -0.0810 & 0.48 \\
$Y_{p}$ & -0.7553 & 29.34 & -0.3379 & 3.84 & -0.2980 & 3.85 \\
$Y_{\delta_{A}}$ & -0.6704 & 14.97 & -0.3563 & 7.08 & -0.3562 & 7.05 \\
$L_{v}$ & -0.0141 & 4.38 & -0.0132 & 0.22 & -0.0133 & 0.22 \\
$L_{p}$ & -0.1607 & 19.54 & -0.2674 & 0.42 & -0.2775 & 0.42 \\
$L_{\delta_{A}}$ & -0.8152 & 15.91 & -3.1147 & 0.22 & -3.5112 & 0.22 \\
$N_{v}$ & 0.0037 & 18.11 & 0.0009 & 3.86 & 0.0008 & 3.83 \\
$N_{p}$ & 0.0907 & 21.00 & 0.0971 & 3.15 & 0.0867 & 3.14 \\
$N_{r}$ & -0.0986 & 10.71 & -0.0724 & 6.32 & -0.0756 & 6.02 \\
$N_{\delta_{A}}$ & 0.3515 & 1.58 & 0.3778 & 1.10 & 0.3785 & 1.10 \\
$N_{\delta_{R}}$ & 0.5079 & 3.39 & 0.2589 & 4.58 & 0.2605 & 4.52 \\
\hline
\end{tabular}

However, one should have in mind that the artificial stabilization introduces modelling errors and therefore there is a greater inaccuracy in those outcomes than in the frequency domain formulation. Nevertheless, the results obtained from both methods are very accurate. This cannot be said about the conventional time domain approach as the resulting parameter estimates have large relative standard deviations and therefore should not be used for modelling purposes.

In order to assess the model behaviour obtained from various OEM approaches, we evaluated the eigenvalues for the model structure given in Eq. (4) and parameter estimates from Table 1. The outcomes are given in Table 2. From this comparison it comes out that the inaccuracies in the time domain parameter estimates affect mostly the lateral phugoid and the spiral mode. The spiral mode is overpredicted what results in too much stability. The same can be observed for the lateral phuigoid and in this case the negative damping is overpredicted what means that the estimated model will diverge faster than the true object. Eigenvalues obtained from the stabilized time domain and frequency domain approaches are very comparable.

Table 2 .

Eigenvalues

\begin{tabular}{|c|c|c|c|}
\hline \multirow{2}{*}{ Method } & \multicolumn{3}{|c|}{ Mode } \\
\cline { 2 - 4 } & Roll & Lateral phugoid & Spiral \\
\hline Time Domain & -0.6413 & $0.1552 \pm 0.4378 \mathrm{i}$ & -0.0986 \\
Stab. Time Domain & -0.6423 & $0.1420 \pm 0.4268 \mathrm{i}$ & -0.0724 \\
Frequency Domain & -0.6442 & $0.1426 \pm 0.4265 \mathrm{i}$ & -0.0756 \\
\hline
\end{tabular}




\section{Conclusions}

In this article, an unstable titltrotor in hover system identification is presented. The output error method was used to obtain stability and control derivatives. Those unknown model parameters were estimated in three ways: by using the conventional time domain approach, by adding artificial stabilization in the time domain formulation and in the frequency domain. Due to the frequency domain limitations, in each case the model was estimated as a linear system.

Problems with states integration have caused that the model parameters estimated in the conventional time domain approach were inaccurate and lead to incorrect values of the aircraft characteristic modes eigenvalues. Adding artificial stabilization matrix allowed us to overcome those numerical difficulties and to obtain estimates with high accuracy regardless the modelling errors introduced in this approach. In the frequency domain, the differential equations were transformed into algebraic equations and therefore it was not necessary to integrate the states. Similarly to the stabilized time domain approach, this formulation also allowed us to obtain the estimates with high accuracy.

To recollect, the unstable tiltrotor was identified with high accuracy by using the stabilized time domain and frequency domain formulations. The conventional time domain approach lead to inaccurate stability and control estimates due to numerical problems.

Manuscript received by Editorial Board, August 22, 2016; final version, January 18, 2017.

\section{References}

[1] J. Kaletka. BO-105 identification results. In Rotorcraft System Identification, AGARD LS-178, AGARD LS-178, Paper 9, November 1991.

[2] R.K. Mehra, R.K. Prasanth, and S. Gopalaswamy. XV-15 tiltrotor flight control system design using model predictive control. In IEEE Aerospace Conference, volume 2, pages 139-148, March 1998. doi: 10.1109/AERO.1998.687905.

[3] R.E. Maine and J.E. Murray. Application of parameter estimation to highly unstable aircraft. Journal of Guidance, Control, and Dynamics, 11(3):213-219, May 1988. doi: 10.2514/3.20296.

[4] S. Weiss, H. Friehmelt, E. Plaetschke, and D. Rohlf. X-31A system identification using singlesurface excitation at high angles of attack. Journal of Aircraft, 33(3):485-490, May 1996. doi: 10.2514/3.46970.

[5] E. Özger. Parameter estimation of highly unstable aircraft assuming linear errors. In AIAA Atmospheric Flight Mechanics Conference, Minneapolis, MN, August 2012. doi: 10.2514/6.20124511.

[6] B. Mettler, T. Kanade, and M. Tischler. System identification modeling of a model-scale helicopter. Technical Report CMU-RI-TR-00-03, Robotics Institute, Pittsburgh, PA, January 2000.

[7] S.K. Kim and D.M. Tilbury. Mathematical modeling and experimental identification of an unmanned helicopter robot with flybar dynamics. Journal of Robotic Systems, 21(3):95-116, March 2004. doi: 10.1002/rob.20002. 
[8] A. Ji and K. Turkoglu. Development of a low-cost experimental quadcopter testbed using an arduino controller for video surveillance. In AIAA Infotech @ Aerospace. AIAA, January 2015. doi: 10.2514/6.2015-0716.

[9] V. Hrishikeshavan, M. Benedict, and I. Chopra. Identification of flight dynamics of a cylcocopter micro air vehicle in hover. Journal of Aircraft, 52(1):116-129, April 2014. doi: 10.2514/1.C032633.

[10] K. Rogowski and R. Maroński. CFD computation of the Savonius rotor. Journal of Theoretical and Applied Mechanics, 53(1):37-45, 2015. doi: 10.15632/jtam-pl.53.1.37.

[11] M.B. Tischler and R.K. Remple. Aircraft and Rotorcraft System Identification. AIAA Education Series. AIAA, Washington, DC, 2 edition, August 2012. doi: 10.2514/4.868207.

[12] B. Etkin. Dynamics of Atmospheric Flight. Dover Publications, Mineola, NY, 2005.

[13] L.A. Zadeh. From circuit theory to system theory. Proceeding of the IRE, 50(5):856-865, May 1962. doi: 10.1109/JRPROC.1962.288302.

[14] T. Söderstörm and P. Stoica. System Identification. Prentice Hall International, New York, 2001.

[15] G.C. Goodwin and R.L. Payne. Dynamic System Identification: Experiment Design and Data Analysis. Academic Press Inc., New York, 1977.

[16] R.V. Jategaonkar. Flight Vehicle System Identification: A Time Domain Methodology. Progess in Astronautics and Aeronautics. AIAA, Reston, VA, 2 edition, 2015. doi: 10.2514/4.102790.

[17] R.E. Maine and K.W. Iliff. Application of parameter estimation to aircraft stability and control: The output-error approach. Technical Report NASA-RP-1168, NASA, Edwards, CA, June 1986.

[18] V. Klein and E.A. Morelli. Aircraft System Identification: Theory and Practice. AIAA Education Series. AIAA, Reston, VA, August 2006.

[19] P. Stoica and R. Moses. Introduction to Spectral Analysis. Prentice Hall, Upper Saddle River, NJ, 2 edition, 2005.

[20] L.R. Rabiner and B. Gold. Theory and Application of Digital Signal Processing. Prentice Hall Inc., Englewood Cliffs, NJ, 1 edition, 1975.

[21] P. Young and R.J. Patton. Frequency domain identification of remotely-piloted helicopter dynamics using frequency-sweep and schroeder-phased test signals. In AIAA Atmospheric Flight Mechanics Conference, Minneapolis,MN, August 1988. AIAA. doi: 10.2514/6.19884349 . 